\title{
Experimental and numerical studies of laminated plates with delamination subjected to compressive loads
}

\author{
Adam Stawiarski* (D) Aleksander Muc ${ }^{a}$ (D), Marek Barski ${ }^{a}$ (D) \\ a Institute of Machine Design, Cracow University of Technology, Cracow, Poland. E-mail: adam.stawiarski@pk.edu.pl, \\ olekmuc@mech.pk.edu.pl, marek.barski@pk.edu.pl
}

* Corresponding author

http://dx.doi.org/10.1590/1679-78256114

\begin{abstract}
In this research, the multilayered composite plates made of glass/epoxy material were experimentally and numerically investigated under compressive loading conditions. The intact and defected structures were analyzed with the use of finite element method. The influence of the thickness and geometrical imperfection level were carried out in the linear buckling analysis. The nonlinear analysis was performed to determine the influence of the delamination length on the buckling behavior of the plate. The numerical results were validated by experiments. Experimental tests were performed for structures having artificial delamination between laminate layers. The buckling behavior was monitored using the nondestructive and noncontact structural vision-based health monitoring system (the digital image correlation (DIC)). The influence of the different delamination behavior during the tests on the compressive load capacity was determined by a detailed analysis of DIC measurements. The $20 \%$ reduction of the compressive load was noticed in the cases with local buckling of delamination.
\end{abstract}

\section{Keywords}

delamination, compressive load, digital image correlation, composite laminate

\section{Graphical Abstract}
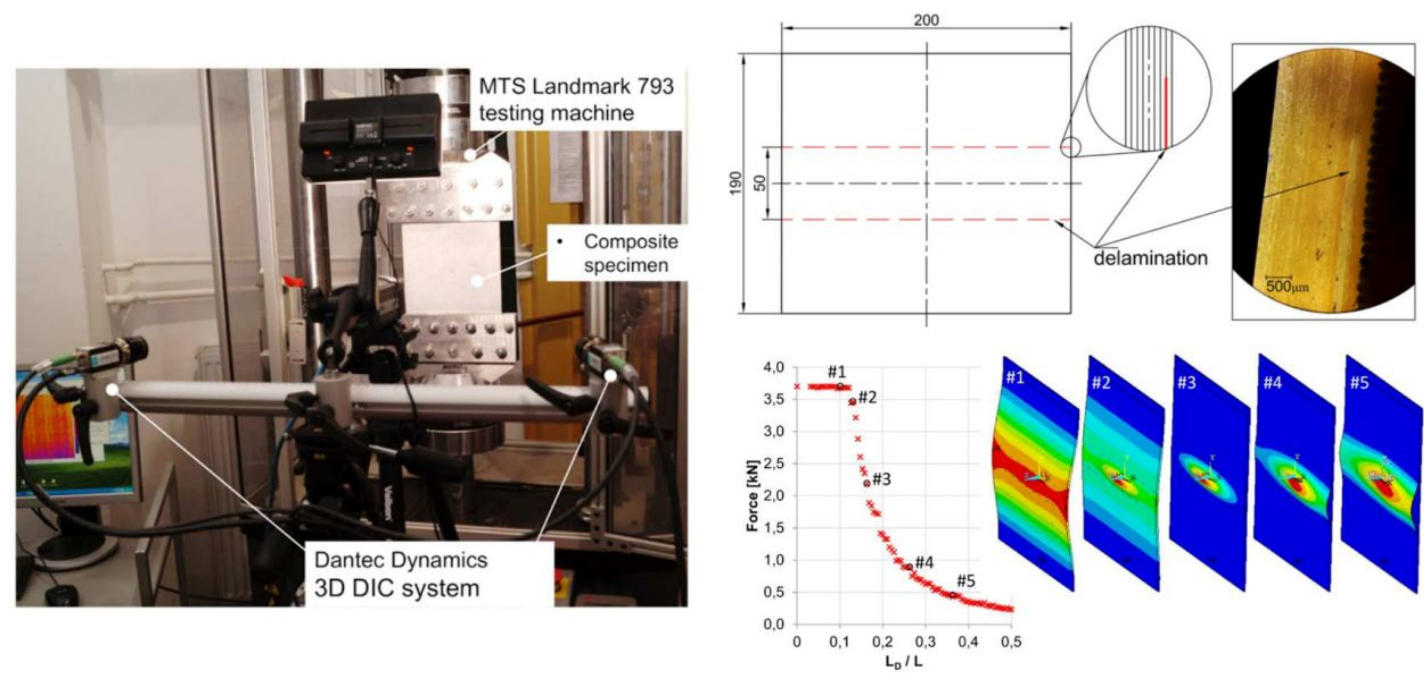


\section{INTRODUCTION}

The accurate assessment of the strength for multilayered structures made of composite materials is difficult because of the great number of possible failure forms. Nowadays, many efforts are dedicated to the development of the Structural Health Monitoring (SHM) systems which allow for permanent or periodical, nondestructive monitoring of a structure during their normal service life. Successful implementation and review of researches were presented by many authors (Doebling et al. (1998), Carden and Fanning (2004), Diamanti and Soutis (2010), Adams (2007), Muc and Stawiarski (2015)). The reliability of the composite elements depends then on the effectiveness and accuracy of the monitoring techniques. In the last decades, a significant number of sensing and monitoring methods were developed with the use of vision-based technology (Ruan et al. (2014), Montesano et al. (2013), Dworakowski et al. (2016)). The non-contact, nondestructive and high precision measurement techniques allow observing and better understanding the failure phenomenon in composite structures.

Buckling is one of the critical failures for layered structures and has essential meaning in aerospace applications. It should be noted, that buckling behavior and critical buckling load depend on various factors (Muc et al. (2018)). Delaminations in composites significantly affect the stability of the structures. The influence of delaminations on the behavior of composite multilayered structures (plates and shells) have been carried out at the Institute of Machine Design for many years. Muc (1995) investigated the influence of delaminations and stacking sequences on buckling loads of compressed cylindrical shells. Then, buckling problems of compressed structures with elliptic delaminations on fatigue failure were studied by Muc (2002). The broader review of works dealing with buckling problems associated with local delaminations was discussed by Muc et al. (2018). Application of the nondestructive methods in the laminated structures with delaminations was also presented by Muc (2011a, 2011b) and Muc and Stawiarski (2012). The mentioned works are an important contribution to the attempt of understanding the failure phenomenon of composites with delaminations under compressive loading conditions which is still analyzed by researchers (Lemanski et al. (2013), Tabiei and Wenlong (2018)). Numerical analysis of buckling and post-buckling behavior of delaminated structures was analyzed with the use of different modeling techniques (Hyo-Jin and Chang-Sun (1997), Hosseini-Toudeshky et al. (2010)). For delaminated structures, it is important to determine the critical delamination length. This length defines delamination size which does not influence buckling load. Gaudenzi (1997) proved delamination length reaches its critical length the buckling load is decreased significantly. Obtained results were confirmed for different composite materials with through the width delamination by Lee and Park (2007) and Damghani et al (2011).

The experimental buckling analysis of the composite structures is difficult because many measurement methods need integration of the sensing equipment with the analyzed structure (Gu and Chattopadhyay (1999)). Recently, the development of non-contact measurement and monitoring methods allow avoiding these difficulties. The Digital Image Correlation (DIC) method was successfully used for measuring the displacement or strain fields of composite laminates. The interlaminar shear strain which could initiate delamination on the free edge of the angle-ply composite was observed with the use of DIC in the micro-scale (Lecornte-Grosbras et al. (2017)). Rhead et al. (2017) applied the DIC system in the analysis of the Compression After Impact (CAl) strength of composite plate with delamination which is crucial to the safety of composite parts in the aerospace industry. The buckling of the delaminated composite laminate after impact damage was considered by Ruan at al. (2014) with the use 3D-DIC system. Riccio at al. (2017) observed delamination growth in composite laminate under compressive loading conditions with the use of DIC and infrared thermography (IRT) techniques. The application of the 3D DIC in determining the size and growth of delaminations was presented by Osman (2018).

The study of the compressive behavior of delaminated structures focuses on great interests because of the necessity of the fulfillment of integrity and safety structural requirements. The dynamic development of the Digital Image Correlation techniques satisfies the increasing demand for accurate and detailed information about deformation and failure of composite structures. Thus, in this paper, the advantages of this technique were used during compressive loading of composite plates having artificial delamination between laminate layers. The finite element analysis modeling of the composite laminate with delamination allows predicting the buckling behavior of the defected composite parts. A detailed experimental analysis supported by the vision-based system was compared with numerical results.

\section{FORMULATION OF THE PROBLEM}

\subsection{Analyzed specimen}

The two composite plates were made of eight layers of glass/epoxy prepreg (TVR 380M12/26\%R) with the dimensions equal to $200 \times 190 \mathrm{~mm}$ having the following mechanical properties: $\mathrm{E} 1=47.12$ [GPa], E2=14.61 [GPa], $v 12=0.09, v 21=0.28, G 12=16.03[\mathrm{GPa}$. The total average thickness of the plates was equal to $2.1 \mathrm{~mm}$. The artificial 
delamination in the middle made of Teflon tape of thickness $0.15 \mathrm{~mm}$ was introduced between the first and second layer (Figure 1).

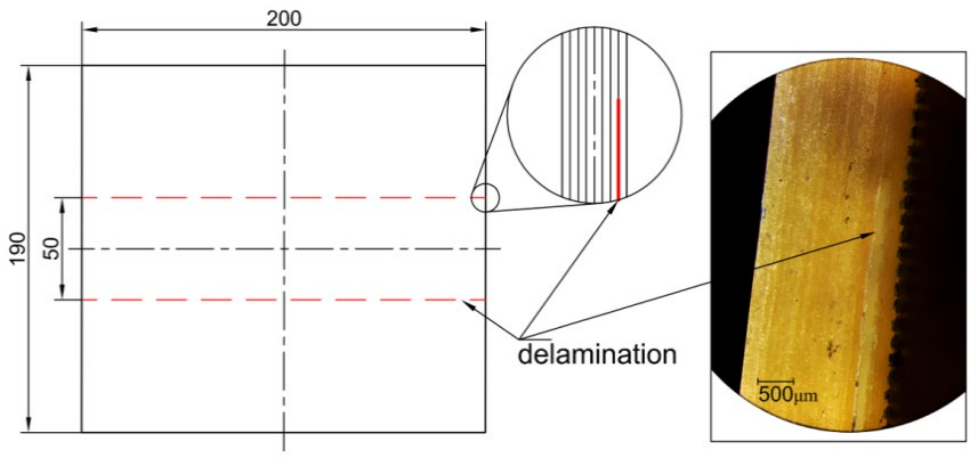

Figure 1: Composite plate with artificial delamination

The angle-ply stacking sequence - $[ \pm 45]_{4}$ is considered. The unsymmetrical layer orientation and the localization of the delamination were chosen to enlarge the possibility of opening of the delamination during the compression of the plates. The specimens were manufactured with the use of the autoclave technique with additional external pressure equal to 4.5 bar. The accurately controlled production process allows for obtaining high quality composite structures with stable dimensions and relatively high fiber volume fraction. Figure $2 a$ demonstrates the thickness field of one of the analyzed plates measured by 3D REVscan scanner dedicated to reverse engineering. It is worth to point out that a controlled manufacturing process caused that the introduced artificial delamination did not cause a local increase of the thickness. Figure $2 \mathrm{~b}$ presents the artificial delamination obtained by the pulse thermography method. Both ends of the plates were tabbed with the use of aluminum alloy plates of dimensions $200 \times 50 \mathrm{~mm}$ and thickness $2 \mathrm{~mm}$ adhesively bonded to the composite specimens. Then, they were mounted to the steel grips by the set of bolts.
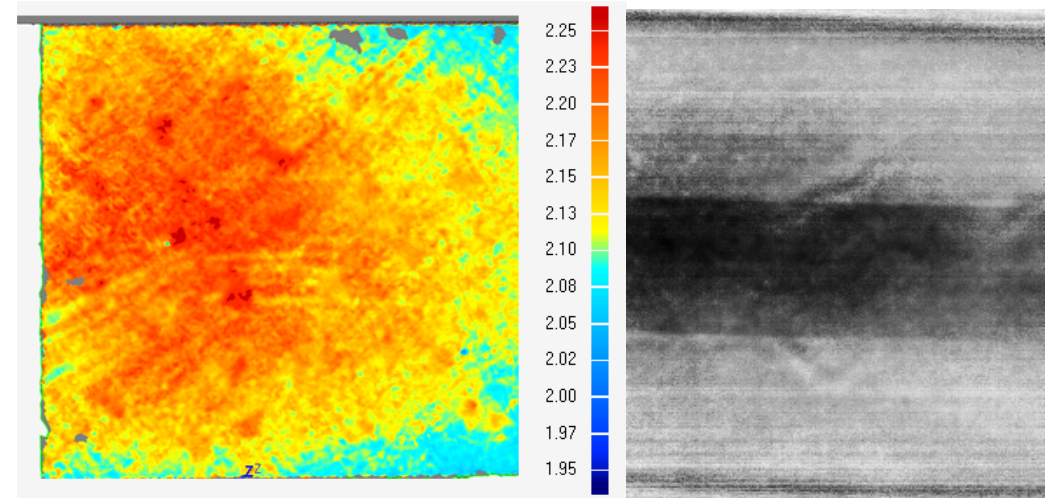

Figure 2: a) The thickness distribution of the composite plate, b) the internal delamination view based on the pulse thermography analysis

\subsection{Numerical FEM model of the plate}

The finite element analysis package ANSYS was carried out in the eigenvalue and nonlinear buckling analysis. The surface models with the use of quadrilateral shell elements having four (shell181) and eight (shell281) nodes per element were compared with the 8-node layered solid-shell element (solsh190). The shell elements have six degrees of freedom (translational and rotational) at each node whereas the solid-shell element possesses the continuum solid element topology with three translational degrees of freedom at each node. The shell elements are often used in the FEM analysis of the composite structures. However, the numerical modeling of the delamination behavior with the use of shell elements with sophisticated techniques (degradation of the stiffness properties of damaged elements, application of coupling of degrees of freedom in the area outside the delamination or introducing the rigid connection by generating constraint equations to relate nodes between shell layers outside the delamination) does not always give satisfying and accurate results. The solid-shell element represents the solid geometry of the structure with the classic layered shell element formulation. In this study the composite plate was modeled by two solid parts (Figure 3b) defining the localization of the delamination. One of the solid parts had thickness equal to a single layer of the laminate with the 
orientation of $45^{\circ}$, and the second solid part had thickness equal to summed thicknesses of the other seven layers and proper fiber orientation definition. The adjacent nodes of the finite elements of mentioned solid parts were merged in the area outside the delamination. Based on this element formulation it is possible to determine the delamination behavior during the deformation. The boundary conditions were applied according to the boundary conditions in the experimental test. Numerical models of the intact composite plate with the use of shell elements and the model of the delaminated plate based on the solid-shell elements were presented in Figure 3.
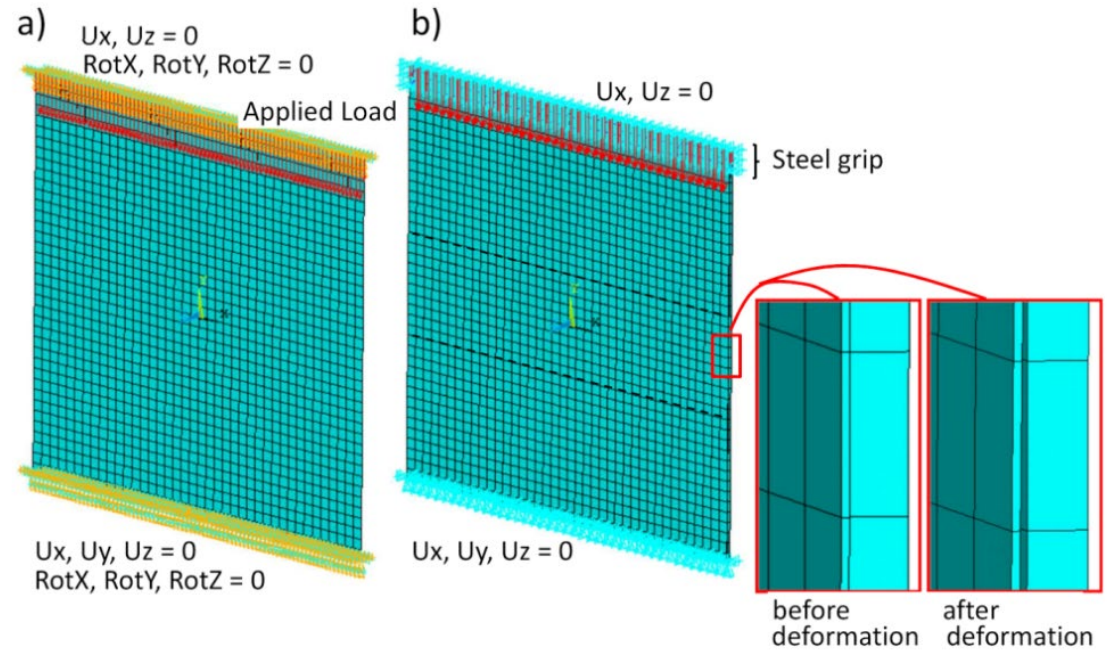

Figure 3: Numerical model of composite plate based on a) shell elements, b) solid-shell elements.

In the solid-shell model, an additional steal grip has been modeled at the top of the structure to enforce the appropriate boundary conditions despite the lack of rotational degrees of freedom. The element size and in consequence, the number of finite elements and nodes were chosen based on the convergence test. The number of the nodes for the shell model was approximately equal to 1600 and for the solid-shell model -5400 . The shell element was applied in the buckling analysis of the intact structures and the validation of the solid-shell element. It is worth pointing that despite the lack of rotational degrees of freedom in the solid-shell model the difference of the results between shell281 and solsh190 elements was lower than $2 \%$.

\subsection{Experimental procedure}

The compressive loading of the composite plates with the delamination was conducted with the use of MTS Landmark 793 testing machine. The displacement controlled loading condition was applied with the velocity equal to $0.1 \mathrm{~mm} / \mathrm{min}$. The maximal value of the vertical displacement (UY) was equal to $0.6 \mathrm{~mm}$ after 360 seconds. The time of the tests was chosen based on the monitoring of the compressive loading capacity measured by the testing machine. The experimental tests were conducted with the use of a digital correlation system (Figure 4).

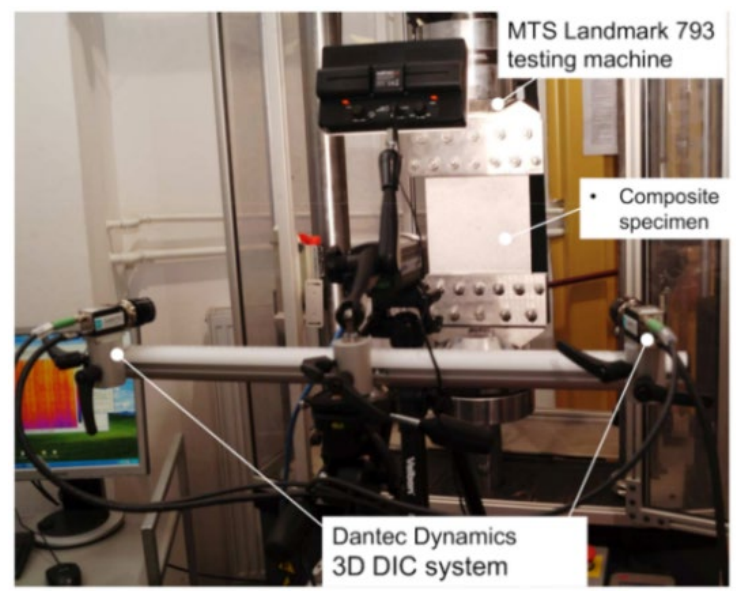

Figure 4: The experimental setup with the digital correlation and the infrared thermography systems. 
The nondestructive and noncontact measuring equipment was used to observe the buckling behavior of delamination. The three-dimensional deformation and displacement field were measured by 3D Dantec Dynamic Q-400 digital image correlation system with the use of two high-resolution cameras. The multi-camera system allows for accurate measurement of out of plane displacement components.

\section{RESULTS}

\subsection{Numerical results of the composite plate without internal defects.}

The eigenvalue and nonlinear buckling analysis were carried out to determine the buckling behavior of the intact plate under force (FC) and displacement (DC) controlled compressive loading conditions. As one can notice in Figure 2a) the real composite plate is characterized by variable thickness. The thickness range depends on the quality of manufacturing methods. In this study, relatively good quality specimens were considered manufactured with the use of autoclave techniques. However, the measured thickness of the plates was in the range from 2.1 to $2.2 \mathrm{~mm}$. Thus, the different value of the plate thickness was also considered in the numerical analysis. The eigenvalue buckling analysis under force controlled loading conditions allows obtaining the buckling load (Fcr) for considered structure thicknesses. The nonlinear buckling behavior was considered with the assumption of geometrical imperfection in the form of the first mode shape. The geometry of the finite element model in the nonlinear analysis is updated according to the displacement result obtained in the linear buckling calculations. Each of the displacement constituents for particular nodes is multiplied by predefined $\lambda$ multiplier describing the level of assumed imperfection. Figure 5 demonstrates the results of the buckling analyzed for considered composite intact plate taking into account all mentioned parameters.

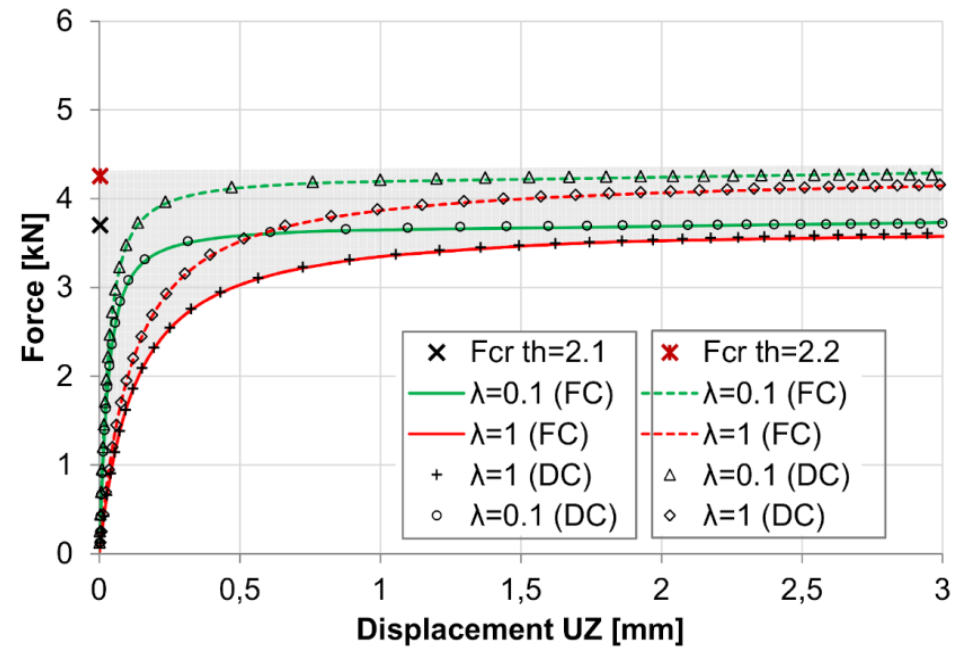

Figure 5: The buckling behavior of the composite intact plate.

The significant influence of the thickness and imperfection level can be noticed. The increase of the thickness by $0.1 \mathrm{~mm}$ causes a $13 \%$ increase of the buckling load - Fcr. The influence of the thickness is also visible in the nonlinear buckling behavior of the plate with imperfection in the form of the first mode shape. The buckling behavior of the structures with different imperfection size has a typical form. However, the results for force controlled (FC) and displacement controlled (DC) loading conditions were almost the same. The level of different type of imperfection is difficult to determine. Taking into account only the variable thickness in the real composite specimen the grey area describes the expected results of the experimental test.

\subsection{The buckling behavior of the composite plate with the delamination.}

The eigenvalue buckling analysis of the composite plate with delamination was performed to determine the influence of the delamination size on the buckling load value. The force controlled loading conditions were used in this analysis due to the convenience of the eigenvalue determination. The linear buckling calculations were also necessary to evaluate the first mode shape which was introduced in the nonlinear analysis as a geometrical imperfection. Figure 6 demonstrates the critical buckling load for different delamination size $\left(L_{D}\right)$ related to the total plate height of $L=190 \mathrm{~mm}$. 

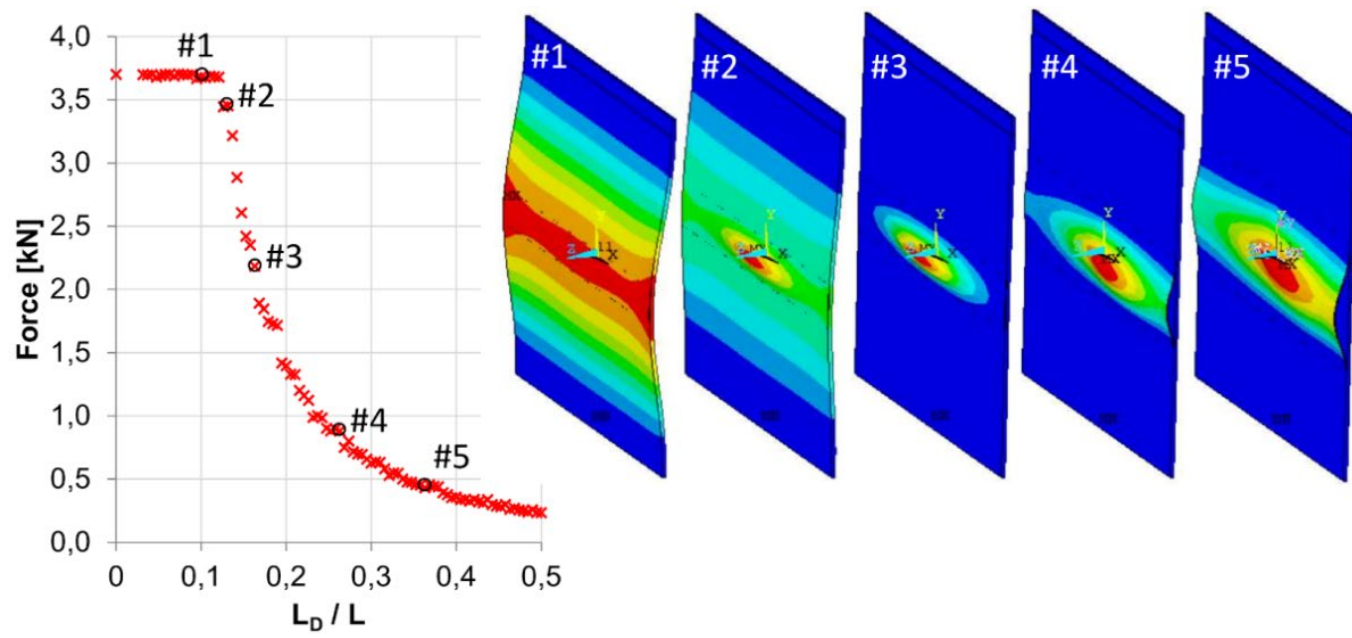

Figure 6: The influence of the delamination size on the buckling load value and the exemplary first mode shapes after linear buckling calculations.

Delamination smaller than $12 \%$ of the analyzed buckling length had no visible influence on the buckling load value. The first mode shape for the small defect is the same as for intact structures. However, the increase of the delamination size $L_{D}$ led to a significant reduction of the buckling load. For example, the delamination size equal to $15 \%$ of the buckling length $\left(L_{D} \approx 30 \mathrm{~mm}\right)$ cause a $42 \%$ reduction of the buckling load. Nevertheless, it is worth noting that the calculated critical buckling load is associated with the loss of stability of the single layer in the area of delamination. To determine the global buckling behavior of the defected plate the nonlinear analysis was performed. Figure 7a demonstrates the influence of the delamination size on the buckling load value taking into account the global buckling behavior of the composite plate.

a)

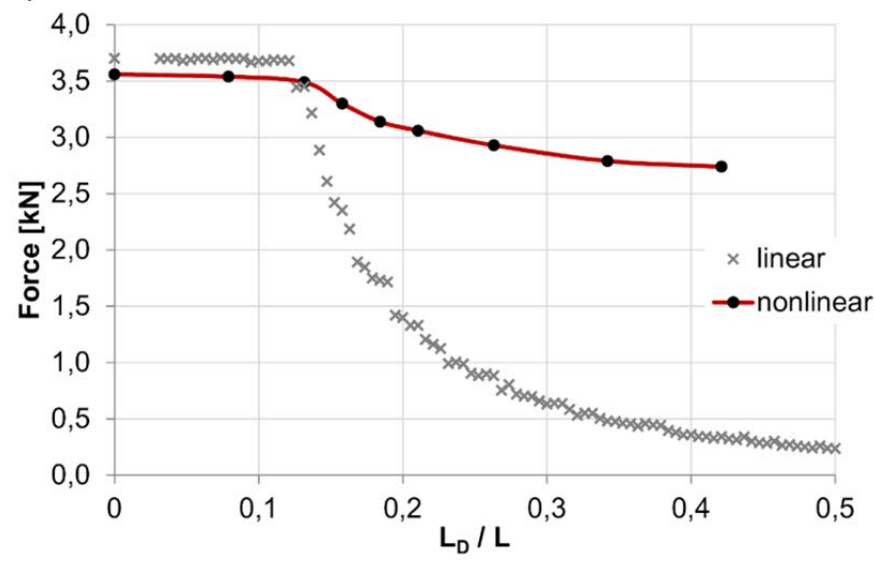

b)

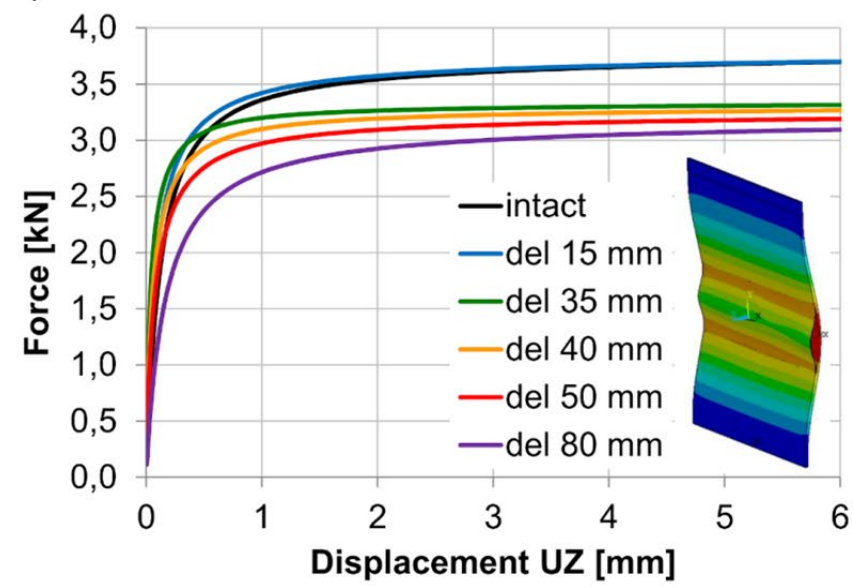

Figure 7: The buckling load value as a function of a) delamination size, b) out of plane displacement.

Similarly to the linear analysis results, a small delamination had no visible influence on the global buckling load. The slightly smaller buckling load value than obtained in the linear analysis can be observed. The reduction of the buckling load for greater defects is also visible in the nonlinear analysis however the level of this reduction was not so huge. For example, delamination size equal to $18 \%$ of the buckling length $\left(L_{D} \approx 35 \mathrm{~mm}\right)$ caused approximately a $10 \%$ reduction of the buckling load value. It means that delamination localized between first and second laminate layers did not significantly reduce the buckling capacity of the composite plate. Figure $7 \mathrm{~b}$ presents the buckling behavior of the composite plate related to the out of plane displacement. The visible influence of the delamination size on the buckling behavior can be noticed. However, the buckling load capacity is finally reduced by $20 \%$ in the case of relatively great defect. The global deformation of the composite plate with the delamination was also demonstrated. The form of the delamination deformation was similar for all considered delamination size cases. 


\subsection{Experimental results of the delaminated composite plate compression.}

As was mentioned the displacement controlled compressive loading condition was applied during the experimental tests of the composite plates with the delamination size equal to $50 \mathrm{~mm}$. The MTS tension machine registered the vertical displacement (UY) and force [kN]. The three failure behaviors were observed during tests and were marked as an S1, S2, and S3 measurements. The S1 measurement was conducted with the use of the first composite plate. In this case, the opening of the delamination was observed from the beginning of the test. The S2 and S3 measurements were carried out on the second plate. During the S2 measurement, the global deformation of the specimens without delamination opening was observed. However, during the second loading of the same specimen (S3), the buckling of the delamination was observed and registered by measurement systems. Figure 8a demonstrates the force characteristics during the loading process for all considered cases. As can be seen, the opening of the delamination was observed at the beginning of the test in the S1 measurement case. The linear increase of the loading force was measured until the level of $3 \mathrm{kN}$. Then the out of plane deformation of the plate caused the limitation of the compressive loading capacity. The schema of the deformation of the plates for considered cases can also be seen in Figure 8a). In the case S2, where the only global form of deformation was observed the smaller influence of delamination to the loading capacity, is registered. The approximately $20 \%$ greater compressive force was measured by a testing machine. The interesting buckling behavior during S3 measurement can be observed on the graph. At the beginning of the test, the global form of deformation was observed and the characteristic of the force was close to the S2 case. However, after vertical displacement equal to $0.3 \mathrm{~mm}$ the sudden buckling of the delamination was noticed. It caused a rapid reduction of the compressive force which characteristic from that moment was almost identical to the S1 case.

a)

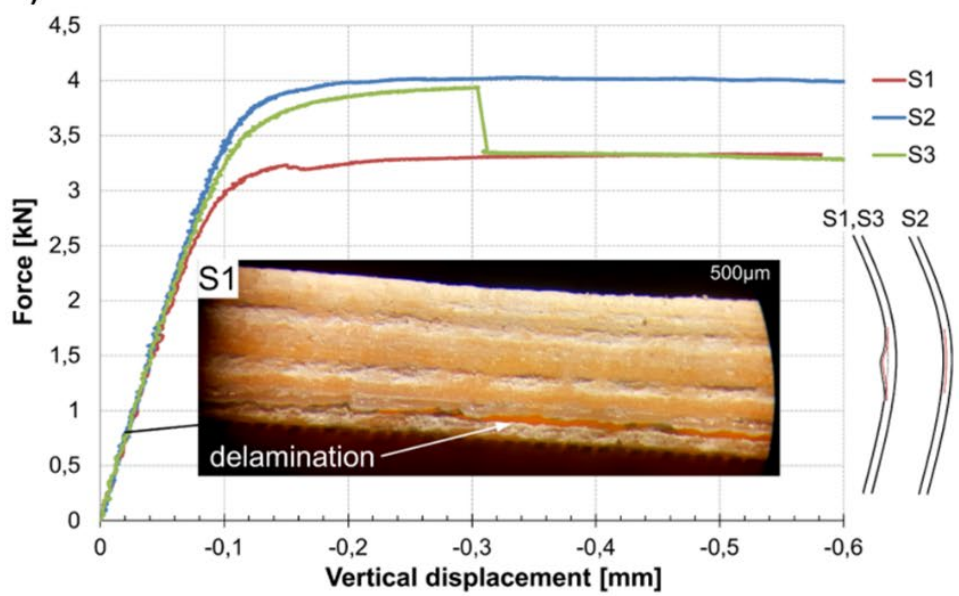

b)

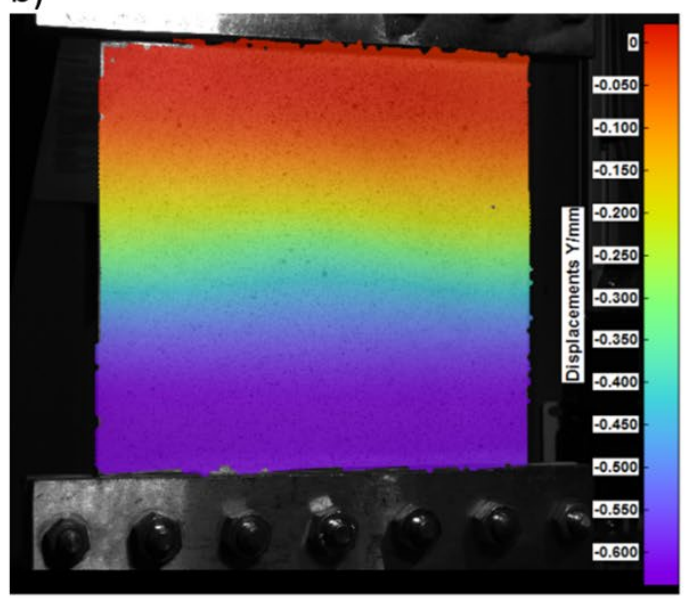

Figure 8: a) The relationship between force $[\mathrm{kN}]$ and vertical displacement $[\mathrm{mm}]$ measured by MTS testing machine, b) the vertical displacement (UY) [mm] at the last step of loading measured by DIC system (S1 case).

The DIC system allows to observe and measure the displacement field at each step of the compressive loading (Figure $8 \mathrm{~b}$ ). For all considered cases the uniform loading condition was provided by applied steel grips. During the tests, the right free edge of the plate was monitored by a standard CCD camera to observe the opening process of the delamination. The out of plane displacement (UZ) measured at three points on the plates (P1-P3) was presented in Figure 9. The images presenting the evolution of the delamination during the compression of the plates are also demonstrated for S1 and S2 cases. In the S1 case, the out of plane displacement measured at the geometrical center of the plate achieves the greatest value. However, it should be emphasized that the DIC system measured the displacement field of the monitored plane and the UZ axis is oriented in the opposite direction to the opening of the delamination. Thus the maximal value at the center of the plate is associated with the global deformation of the plate. The buckling of the delamination is clearly visible at points P1 and P3 measured near the free edges of the specimen. The out of plane displacement in P1 and P3 points are lower than in the center because the opening of the delamination occurs in the opposite direction to the global deformation of the plate. The buckling of the delamination was observed at the right edge by CCD camera. The interesting effect of higher buckling mode can be noticed at the beginning of the deformation process (S1 - time $145 \mathrm{~s}$ in Figure 9). One can notice two half waves formed by the external laminate layer. The displacement UZ path measured in point $\mathrm{P} 3$ is different than measured at the second free edge of the plate. 
However, after approximately 160 seconds of the compressive loading, the sudden change of the buckling mode was observed ( $\mathrm{S} 1$ - time $180 \mathrm{~s}$ ). From this moment the displacement path was parallel to the path measured in point P1.

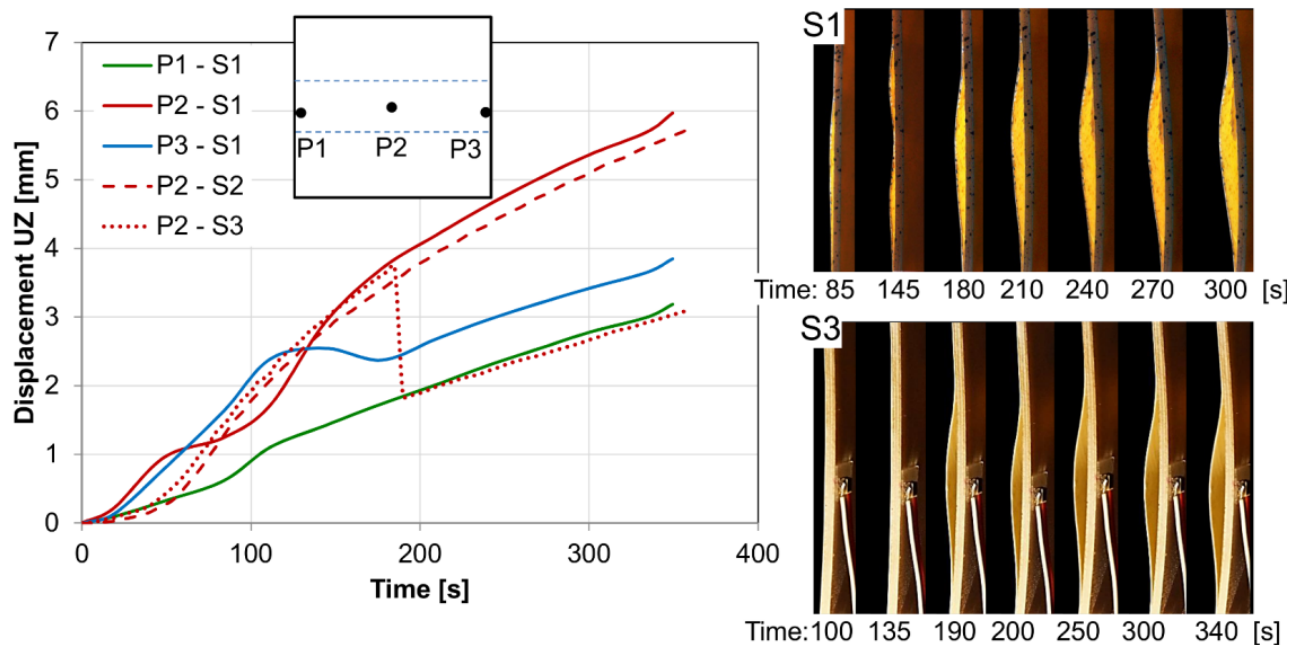

Figure 9: The out of plane displacement UZ [mm] for specimens S1 and S2 at points P1- P2 and the evolution of the delamination observed at one free edge of the plates.

One can notice the unsymmetrical behavior of the delamination in the $\mathrm{S} 1$ case. This situation could be caused by many factors like different imperfections associated with artificial delamination and the composite plate or some inhomogeneity in the area of delamination obtained in the manufacturing process. Nevertheless, similar problems with composite laminates can also be observed in the cases of real structures with defects. Moreover, the typical failure forms in composite materials have a stochastic character and the accurate prognosis of the damage evolution is difficult. Thus, even such unsymmetrical behavior observed in the experimental test should be taken into account. In the S2 and S3 measurements, the symmetrical behavior was observed so only middle point P2 was demonstrated in Figure 9. The displacement UZ measured at the center of the specimen for the S2 case is similar to the analogical measurement in the S1 case. It proves the small influence of delamination in this area measured in the S1 case. Until time equal to approximately 175 seconds the displacement measured at $\mathrm{P} 2$ point for all considered cases are similar and is associated with global deformation of the plate. However, in the S3 the sudden buckling of the delamination was observed what caused the decrease of force and out of plane displacement. The rapid decrease of displacement UZ measured by the DIC system is connected with the direction of the delamination opening opposite to the global deformation of the plate. It is worth pointing out that further time characteristic of the P2 displacement UZ is almost identical to the case S1 where the opening of the delamination was observed from the beginning of the test. The evolution of the delamination for the S3 case observed by the CCD camera at the right edge of the plate was also demonstrated in Figure 9. It is clearly visible that after buckling of delamination in $\mathrm{S} 3$ measurement the level of opening of the defect is similar in both presented cases (S1 and S3).

The Digital Image Correlation system makes a possible detailed analysis of the buckling behavior by permanent monitoring of the displacement field components. Figure 10 demonstrates the out of plane displacement distribution on the surface of the specimen after the last step of the loading. The displacement UZ horizontal $(\mathrm{H} 1)$ and three vertical (V1, V2, and V3) profiles were also demonstrated. 
a)

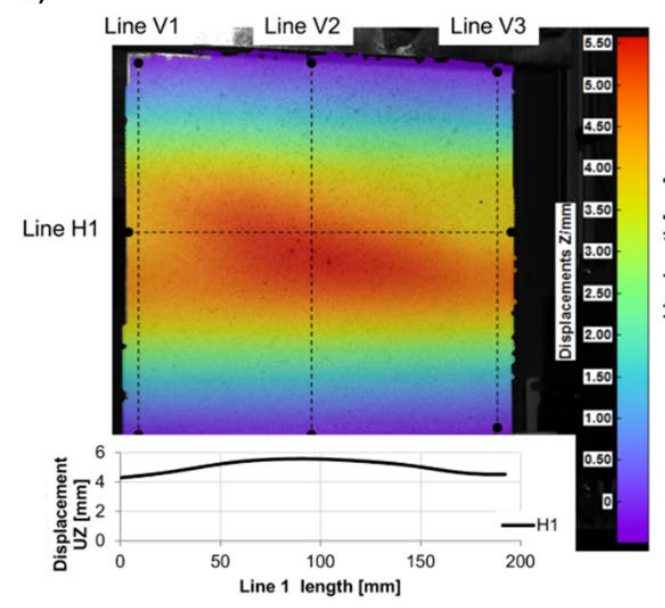

b)

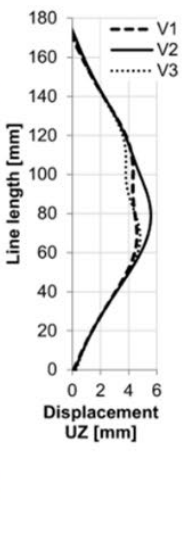

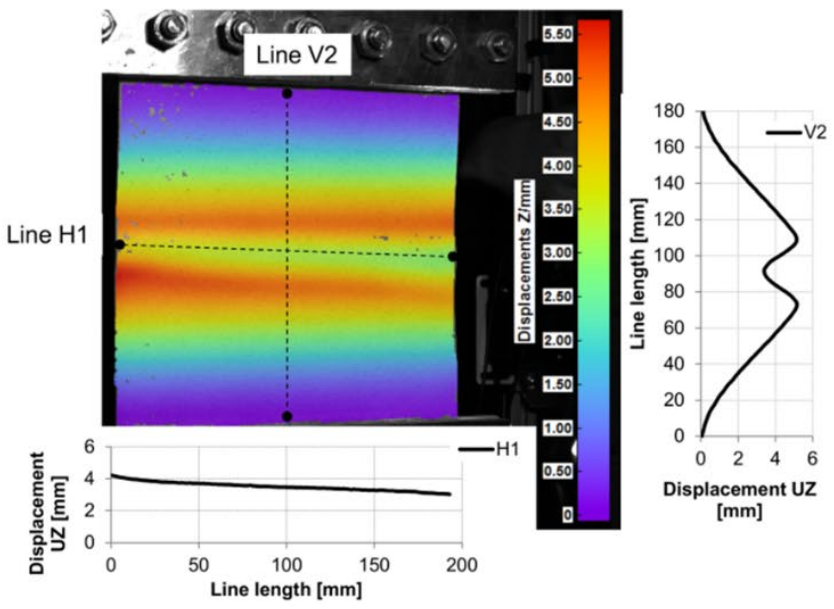

Figure 10: The displacement UZ field on the measured plane for measurement a) S1 and b) S2 after the last step of loading.

The greatest value of the displacement UZ in the S1 measurement case (Figure 10a) is localized at the center of the specimen as was presented earlier at point P2. The displacement profiles on the vertical lines demonstrate the influence of the delamination at the edges of the plate. The displacement UZ measured at the line V2 is smooth and continuous at the center of the plate. It indicates the relatively small influence of the delamination on the global deformation of the specimen in this area. The influence of delamination is greater near the free edges of the plates. The shape of the buckled delamination is clearly seen in profiles V1 and V3. In the measurement S3, the buckling of the delamination through the entire width of the plate was observed (Figure 10b). The displacement field measured on profile V2 demonstrates the opening of delamination in the central area of the plate. The displacement field observed in this test is similar to the out of plane displacement in the numerical analysis presented in Figure $7 \mathrm{~b}$. However, one can notice in the displacement profile $\mathrm{H} 1$ that the different level of the opening is observed on the free edges of the specimen. Figure 11 demonstrates the process of delamination opening during the tests measured on vertical profiles for all considered cases. Figure 11a presents the three profiles analyzed in the S1 measurement. The differences between displacement measured on the particular profiles are small at the beginning of the test. One can notice the increasing differences between displacement UZ measured on the Line V2 localized at the center of the plate and on Lines V1 and V3 placed at the edges of the plate. The mentioned earlier buckling mode change at the right free edge of the S1 case can also be noticed. Figure 11b demonstrates the progressive global deformation of the plate without the visible influence of the artificial delamination. The buckling of the delamination under compressive loading condition is clearly seen in Fig. 11c where the displacement UZ for profile V2 in the S3 case is demonstrated.

a)

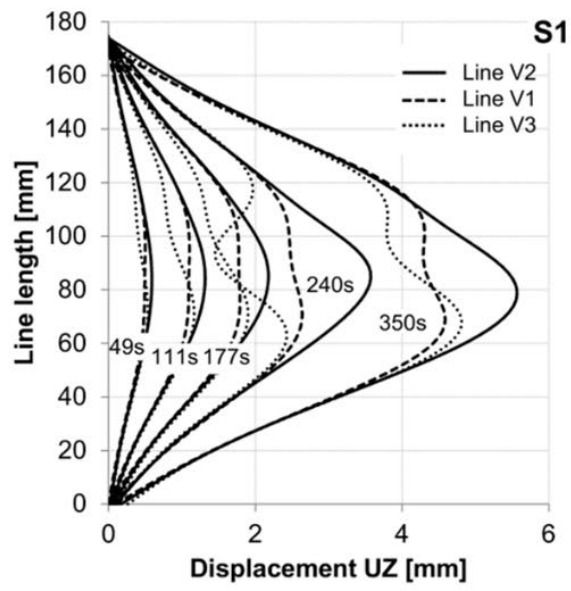

b)

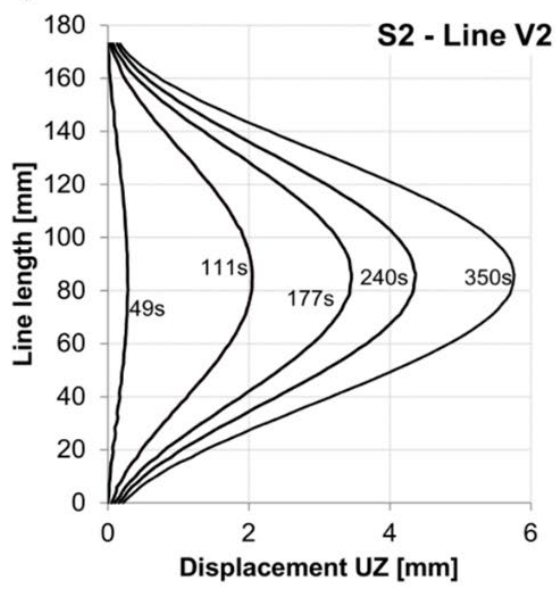

c)

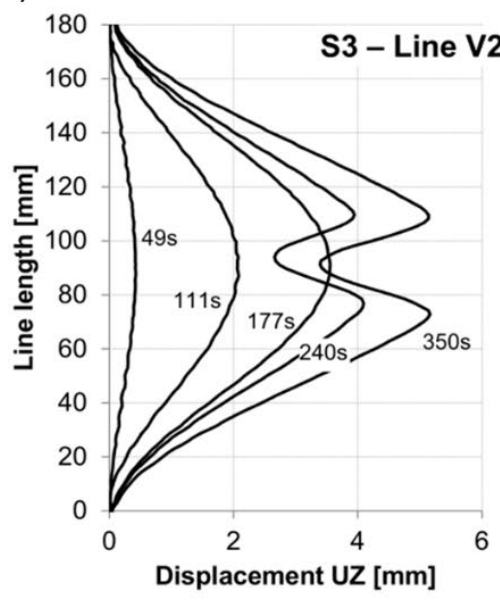

Figure 11: The displacement UZ measured on vertical profiles for a) S1, b) S2 and c) S3 cases. 
The direct comparison of the displacement UZ measured on vertical and horizontal profiles after the last step of loading is demonstrated in Figure 12. The measurement S2 presents the global form of the specimen deformation without the essential influence of delamination is (Figure 12a).

a)

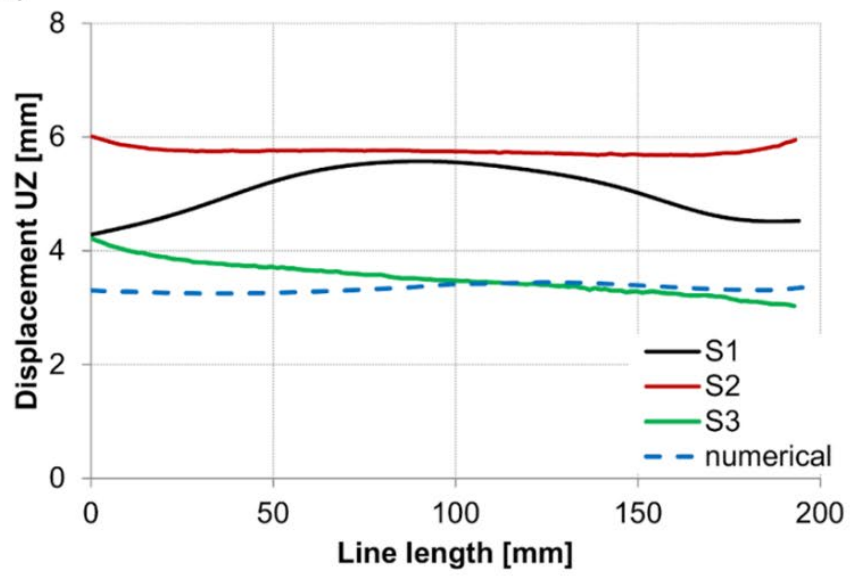

b)

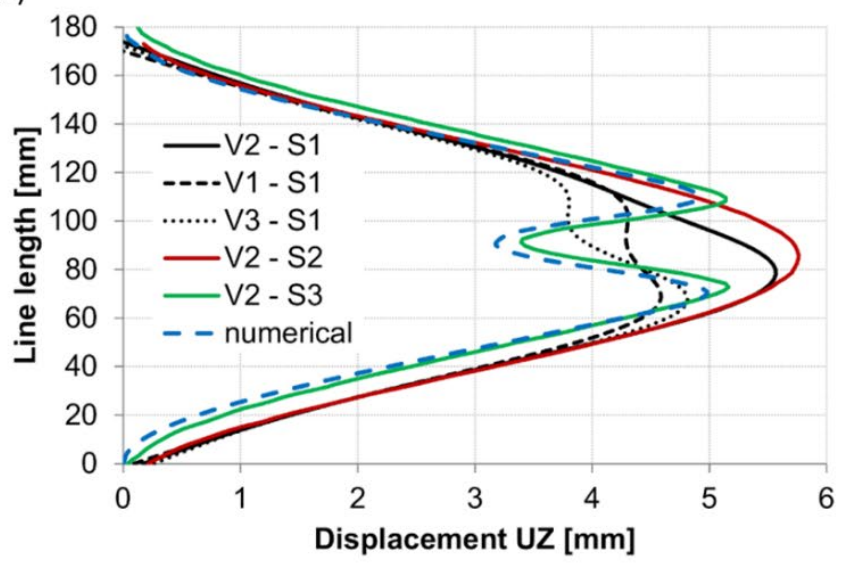

Figure 12: The displacement UZ measured on a) horizontal and b) vertical profiles after the last step of loading.

As was noticed earlier the opening of the delamination for S1 case was noticed at the edges of the plate. For the S3 measurement, the level of the out of plane displacement at the left free edge is almost identical to the S1 case. However, the opening of the delamination at the right edge of the plate is visible greater. The comparison of the vertical profiles (Figure 12b) shows the similar global deformation of the plates with differences in the area of delamination. The results of the numerical nonlinear analysis of the composite plate with delamination size equal to $50 \mathrm{~mm}$ is comparable with the S3 case. The influence of the delamination behavior on the compressive load capacity is demonstrated in Figure 13. Despite considering the same delamination size for all analyzed plates, the significant differences in load capacity are measured. The gray area was repeated from Figure 5 and describes the numerical results of the intact composite plate with different levels of imperfection and thickness. The compressive force measured by MTS testing machine in the case of S2 where the global deformation of the plate was observed is equal to $4 \mathrm{kN}$. The whole equilibrium path is in the gray area from the numerical results which means the negligible influence of the delamination in the case of the global buckling of the defected structure without visible delamination opening. In the case S1 where the opening of the delamination starting from the beginning of the test, the maximal value of the force is equal to $3.3 \mathrm{kN}$. It means almost a $20 \%$ decrease in the compressive load capacity caused by stiffness reduction of the plate. The characteristic of the compressive force for the S3 case was similar to the S2 measurement until the buckling of the delamination was observed. A similar load decrease was measured. The good agreement of the buckling load reduction between numerical and experimental tests was observed. The equilibrium path of the numerical results was similar to the S3 case at the beginning of the compressive loading. However, because of the assumed geometrical imperfection, the sudden delamination buckling was not observed in FEM analysis. The reduction of the out of plane displacement after delamination buckling is associated with the direction of the delamination opening and the fact that the displacements field UZ demonstrates the behavior of the external surface of the plates. 


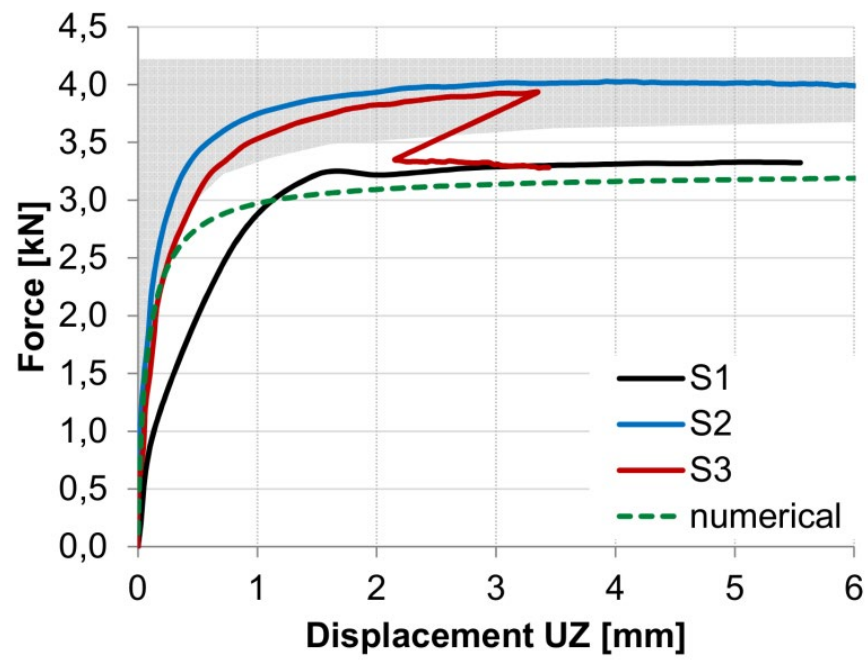

Figure 13: The force-displacement UZ curves for considered cases.

\section{CONCLUSION}

The different failure form of laminated plates having artificial delamination was studied in this paper. The experimental results of the compression tests present different possible damage evolution forms which affect the compressive strength of the plate. The coupled noncontact measurement methods make possible detailed analysis of the failure form evolution. Digital Image Correlation system allows observing the behavior of defected structures during the compressive tests. Despite testing geometrically the same specimens, three different damage evolution was observed. The global deformation of the specimen without opening of the delamination caused the higher level of the compressive force, however when the delamination opening occurred the load capacity significantly decreased. Moreover, the level of the load decrease was similar for the case when the delamination was opening from the beginning of the test and for the case when the sudden buckling of the delamination was noticed. For all analyzed specimens the reduction of the compressive load capacity was approximately equal to $20 \%$. Thus, one can notice that the different behavior of the delamination did not influence the achieved loading level. The experimental results were compared with the numerical one. A good agreement was observed in the case of buckling load reduction. The equilibrium path was slightly different because of the assumption of geometrical imperfection in the form of the first mode shape. However, the finite element analysis allows for observing the buckling behavior of the composite plate with the delamination. The influence of the delamination size on the buckling load reduction was determined. It was shown that a relatively small defect did not change the buckling load. The maximal buckling load reduction achieved for greater defects was approximately the same obtained in the experimental tests.

Author's Contribuitions: Conceptualization, A Muc; Formal analysis, Writing - Original Draft, Visualization, A Stawiarski; Investigation, M Barski.

Editor: Marcílio Alves.

\section{References}

Adams, D.E., (2007). Health Monitoring of Structural Materials and Components, Methods and Applications, John Wiley \& Sons Ltd.

Carden, E.P., Fanning, P., (2004). Vibration based condition monitoring: a review. Structural Heatlh Monitoring 3:355-377.

Damghani,D.,Kenned, D., Featherston, C., (2011). Critical buckling of delaminated composite plates using exact stiffness analysis. Computers and Structures 89:1286-1294.

Diamanti, K., Soutis, C., (2010). Structural health monitoring techniques for aircraft composite structures. Progress in Aerospace Science 46:342-352. 
Doebling, S.W., Farrar, C.R., Prime, M.B., (1998). A summary review of vibration-based damage identification methods. Shock and Vibration Digest 30:91-105.

Dworakowski, Z., Kohut, P., Gallina, A., Holak, K., Uhl, T., (2016). Vision-based algorithms for damage detection and localization in structural health monitoring. Structural Control and Health Monitoring 23:23-50.

Gaudenzi, P. (1997). On delamination buckling of composite laminates under compressive loading. Composite Structures 39:21-30.

Gu, H., Chattopadhyay, A., (1999). An experimental investigation of delamination buckling and postbuckling of composite laminates. Composite Science and Technology 59:903-910.

Hosseini-Toudeshky, H., Hosseini, S., Mohammadi, B., (2010). Delamination buckling growth in laminated composites using layerwise-interface element. Composite Structures 92: 1846-1856.

Hyo-Jin, K., Chang-Sun, H., (1997). Buckling and postbuckling behavior of composite laminates with a delamination. Composite Science and Technology 57:557-564

Lecornte-Grosbras, P., Paluch, B., Brieu, M., De Saxcé, G., Sabatier, L., (2017). Interlaminar shear strain measurement on angleply laminate free edge using digital image correlation. Composites Parta A: Applied Science and Manufacturing 40:1911-1920.

Lee, S-Y., Park, D-Y., (2007). Buckling analysis of laminated composite plates containing delaminations using the enhanced assumed strain solid element. International Journal of Solids and Structures 44:8006-8027.

Lemanski, S.L., Wang, J., Sutcliffe, M.P.F., Potter, K.D., Wisnom, M.R., (2013). Modelling failure of composite specimens with defects under compression loading. Composites Part A: Applied Science and Manufacturing 48: 26-36.

Montesano, J., Fawaz, Z., Bougherara, H., (2013). Use of infrared thermography to investigate the fatigue behavior of a carbon fiber reinforced polymer composite. Composite Structures 97: 76-83.

Muc, A., (2011a), Modeling damage in cylindrical shells using elastic wave-based techniques, Proceeding of the18th International Conference on Composite Materials ICCM18, Jeju, Korea.

Muc, A., (2011b), SHM of composite cylindrical multilayered shells with delaminations, Proceedings of the IUTAM Symposium on Dynamics Modeling and Interaction Control in Virtual and Real Environments, Budapest, Hungary

Muc, A., Chwał, M., Barski, M., (2018). Remarks on experimental and theoretical investigations of buckling loads for laminated plated and shell structures. Composite Structures 203:861-874.

Muc, A., Stawiarski, A., (2012). Wave propagation in composite multilayered structures with delaminations. Mechanics of Composite Materials 48: 101-106

Muc, A., Stawiarski, A., (2015). Location of delaminations in curved laminated panels, Composite Structures 133: 652-658.

Muc,A.,(1995). Interlaminar failure and buckling of doubly-curved laminated composite shells. Mechanics of Composite Materials 31: 238-246.

Muc,A.,(2002). A fuzzy set approach to interlaminar cracks simulation problems. International Journal of Fatigue 24: $419-427$.

Osman, Z.A., (2018). The use of Digital Image Correlation to monitor delaminations in composite structures. Doctoral dissertation, University of Surrey

Rhead, A.T., Butler, R., Hunt, G.W., (2017). Compressive strength of composite laminates with delamination-induced interaction of panel and sublaminate buckling modes. Composite Structures 171:326-334.

Riccio, A., Cristiano, R., Mezzacapo, G., Zarrelli, M., Toscano, C., (2017). Experimental investigation of delamination growth in composite laminates under a compressive load. Advances in Materials Science and Engineering 3431093:1-17.

Ruan, J.T., Aymerich, F., Tong, J.W., Wang, Z.Y., (2014). Optical evaluation on delamination buckling of composite laminate with impact damage. Advances in Materials Science and Engineering 390965: 1-9.

Tabiei, A., Wenlong, Z., (2018). Composite laminate delamination simulation and experiment: a review of recent development. Applied Mechanics Reviews 70:1-23. 